\title{
Storage Subsystem for Multimedia Server
}

\section{Katsuhiko NISHIKAWA, Kohichi SHIGA, and Kazuhisa SEKI}

1) Fujitsu Laboratories Ltd., 1-1, Kamikodanaka 4, Nakahara-ku, Kawasaki 211-88, Japan

2) Fujitsu Ltd., 1-1 Kamikodanaka 4, Nakahara-ku, Kawasaki 211-88, Japan

\begin{abstract}
We have studied storage subsystems for multimedia servers in application systems that require huge storage capacities. The importance of multimedia servers has grown recently due to the increasing popularity of online services such as home shopping and video-on-demand. In this paper, we describe an architecture suitable for high-speed transmission of multimedia data. We also explain the characteristics of our Multimedia Stream Adapter (MSA), which we developed based on this architecture. Using this technology, we have built the Fujitsu Media Server ${ }^{* 1}$, which is capable of providing the most cost-efficient multimedia information system available.
\end{abstract}

Key words: VOD, ATM, MPEG, stream

\section{INTRODUCTION}

High-quality image coding techniques such as MPEG2 (Moving Picture Experts Group) and high-speed networks such as ATM (Asynchronous Transfer Mode) have all been evolving steadily. At the same time, highperformance personal computers that easily process digital images are commonplace. For these reasons, video-ondemand and other real-time interactive multimedia services capable of handling large amounts of image data are growing quickly. These services require multimedia servers that can store huge amounts of multimedia data and transmit that data at high speed.

We have developed an architecture suitable for largescale multimedia information services on public networks and corporate intranets. We have also developed a multimedia stream adapter (MSA) board based on the architecture. Using new techniques, the board enables high-speed image data transmission, which is the most important requirement for multimedia servers.

This paper describes the features of the storage subsystem for the Fujitsu Media Server. This multimedia server has attained the highest level of performance and

\footnotetext{
"The product name of Fujitsu's media server system.
}

cost efficiency by using the MSA board.

\section{ARCHITECTURE OF THE MULTIMEDIA SERVER}

The basic function of a multimedia server is to transmit on demand multimedia data stored on a hard disk or other storage unit over a network to user. Multimedia servers designed for medium- to large-scale services should be scalable to meet various service requirements and should provide many concurrent streams so that service costs can be minimized.

Conventional multimedia server configurations include the use of the appropriate software on multiple personal computers or workstations, the assignment of stream transmission functions to the nodes of a parallel computer, and dedicated machines. All these approaches entail high costs. An architecture that provides sufficient scalability, many concurrent streams, and a high cost-efficiency is needed.

To this end, we developed a dedicated machine that has high concurrent stream transmission capabilities for use as the nucleus stream transmission section of a multimedia server. The remainder of the server consists of general-purpose units. Based on this architecture, an MSA board has been developed for the stream transmission section, and we developed a video storage subsystem incorporating the MSA board as the nucleus of the Fujitsu Media Server.

\section{CONFIGURATION OF THE FUIITSU MEDIA SERVER}

The Fujitsu Media Server system includes a management server that manages the entire system, a video storage subsystem (VSS) that stores and transmits image data, a content server that stores video data in the VSS, and a client, which is connected through an ATM switch and displays video (Figure 1), The user selects the desired 


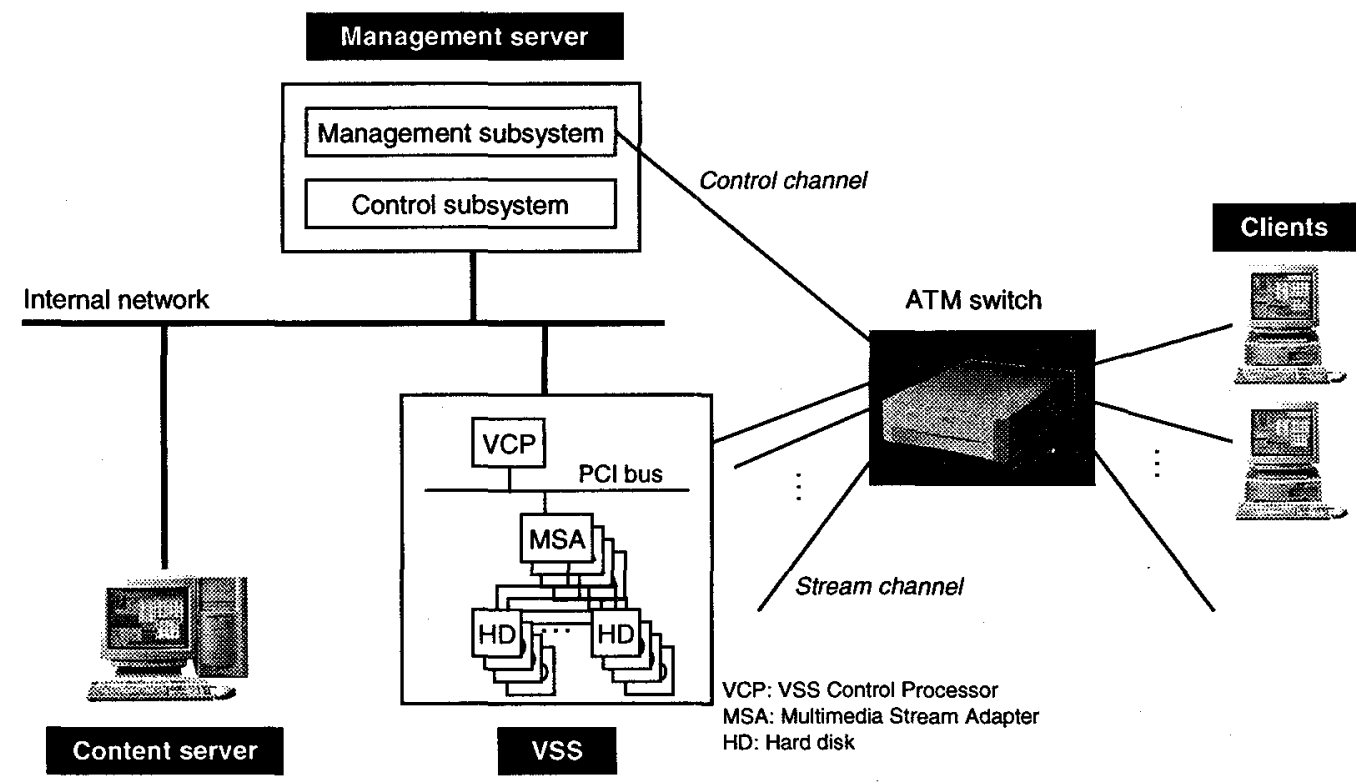

Figure1. Configuration of the Fujitsu Media Server

video data interactively through the client. A stream playback request is sent through the control subsystem on the management server to the VSS, which transmits video and other multimedia data as a stream. The VSS is a multimedia disk unit containing MSA boards and disks. The MSA and the VSS, which are the core components of this system, are described in following sections.

\section{A. MSA}

The high-performance, compact MSA board was developed based on the stream transmission board used in the AIMS (ATM-based Interactive Multimedia Server),

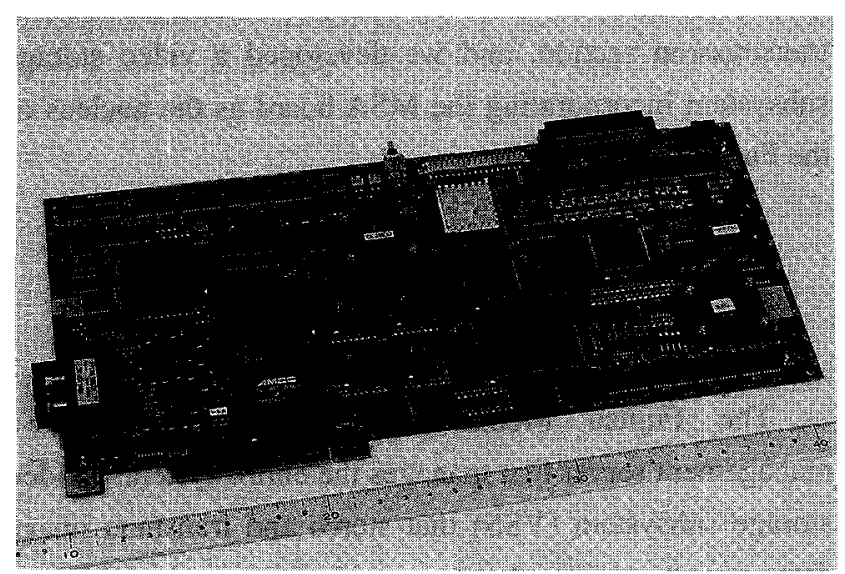

Figure 2. MSA board which was produced as a prototype in 1995[1]. Figures 2 and 3 show a photograph and a block diagram of the MSA. As shown in Figure 3, the MSA board has a disk controller, an ATM controller that assembles and disassembles ATM cells, data memory that temporarily holds multimedia data, and a data memory controller that controls data memory access from the disk and the ATM controller. It also has a CPU that controls the MSA board, a CPU program memory, and a PCI (Peripheral Component Interface) bus interface that interfaces with the VSS control processor, or VCP.

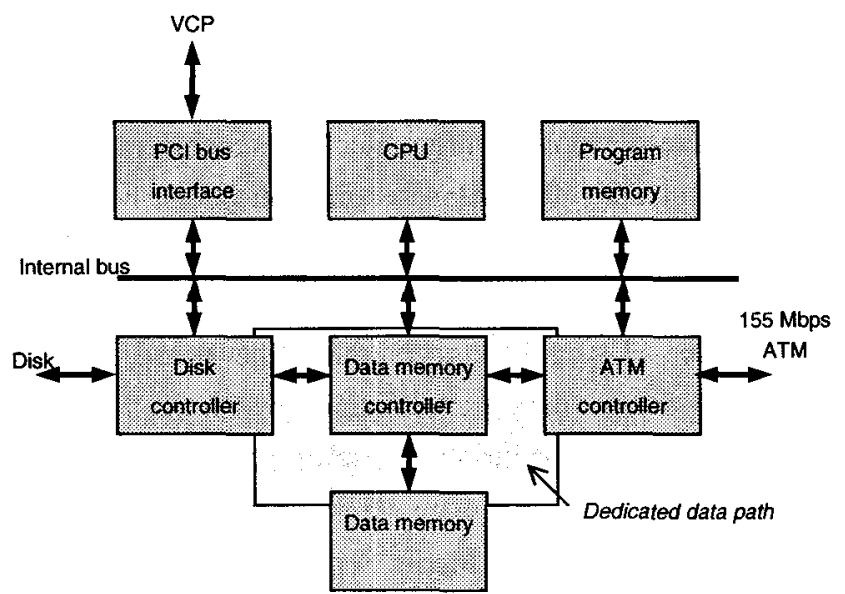

Figure 3. MSA block diagram 
The MSA board has the following features:

1) Localized stream transmission processing

As described in Section II, conventional architectures have the following problems:

- The data stream passes through a system bus.

- A host CPU controls the data transfer from disks to a network.

This means that the performance of stream transmission is affected by bus loads and CPU loads, so it is difficult to achieve high-speed stream transmission. To solve these problems, we designed a new architecture in which disk and network interfaces are connected with a dedicated data path. With this architecture, the MSA board transmits a stream utilizing the on-board CPU. When a stream is transmitted, data read from a disk passes only through the MSA board. Because the processing is executed only in the MSA board, a high stream transmission capability can be maintained, irrespective of the VCP load on the VSS or PCI bus.

2) Retransmission control function

On corporate intranets, parts of streams may be lost due to network congestion. On the other hand, TCP/IP (Transmission Control Protocol/Internet Protocol) and other handshaking protocols increase the CPU load and thus degrade stream transmission performance. To solve this problem, we have implemented a new protocol for retransmission of lost data. The protocol is placed on UDP/IP (User Datagram Protocol/Internet Protocol) and doesn't use handshaking. Retransmission using this protocol works as follows:

(1) A server transmits a packet with a sequence number which increases for each transmission by one.

(2) When a receiver detects discontinuity of sequence number, the receiver transmits a packet that requests retransmission of the lost packets.

(3) The server transmits the requested packets.

Because the packets with which a receiver requests retransmission will be transmitted only when packets are lost, this protocol is lighter than other ones that always use the handshaking mechanism.

\section{3) Efficient disk access}

Multimedia data is divided and written on two or more disks to enable high-speed access. Data on different disks is accessed through different time slots so that the access time of each disk is concealed. Data is continuously read from the disk controller, maximizing the number of concurrent streams (Figure 4).

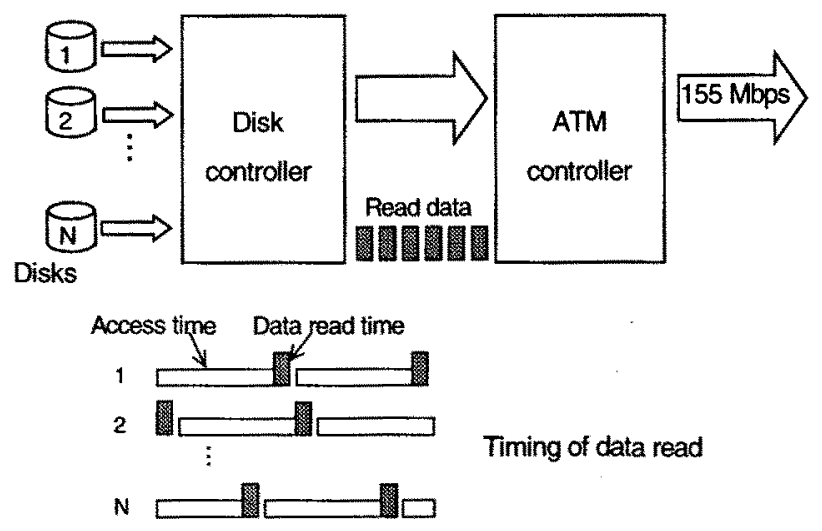

Figure 4. Optimized disk access

4) Checksum calculation function

The data memory controller controls access to data memory and adds a checksum to data transferred from the disk controller. The checksum function enables highspeed stream transmission when UDP/IP or another Internet protocol that requires checksum calculations is used.

\section{5) Software RAID function}

One disk connected to the MSA board is used as a parity disk. When multimedia data is written, the exclusive-OR value of the data on the other disks is calculated and stored on the parity disk. If a disk crashes, its data can be reconstructed from the exclusive-OR of the data on the other disks. In this way, data can be quickly recovered if a disk failure occurs.

With these features, the MSA board developed for corporate intranets enables concurrent transmission of 11 streams at $6 \mathrm{Mbps}$.

\section{B. VSS}

The VSS is a multimedia disk unit containing the MSA board and disks. The VSS transmits a stream in response to a control command issued by the management server. Table 1 lists the major specifications of the VSS, and Figure 5 shows what it looks like. 
Table 1. Major specifications of the VSS

\begin{tabular}{c|c}
\hline \hline Item & Specification \\
\hline $\begin{array}{c}\text { Number of } \\
\text { concurrent streams }\end{array}$ & $\begin{array}{c}\text { UDP/IP over ATM: 44 streams } \\
\text { (6 Mbps) }\end{array}$ \\
\hline Storage capacity & $\begin{array}{c}\text { Up to 172 GB } \\
\text { (4.3 GB disk mounted) }\end{array}$ \\
\hline Number of MSAs & Up to four \\
\hline Data channel & - UDP/IP over ATM \\
protocol & - MPEG over ATM (projected) \\
\hline ATM interface & STM-1/OC-3c \\
\hline \hline
\end{tabular}

The VSS has the following features:

\section{1) Many concurrent streams}

Since the stream processing is executed only in the MSA board, a high stream transmission performance can be maintained, unaffected by the VCP processing or data transfer of the PCI bus.

\section{2) High cost efficiency}

The system uses the MSA board as the stream transmission section, resulting in a low cost per stream. By using the PCI bus common to many personal computers, general-purpose products can be used. For these reasons, the overall system cost is reduced.

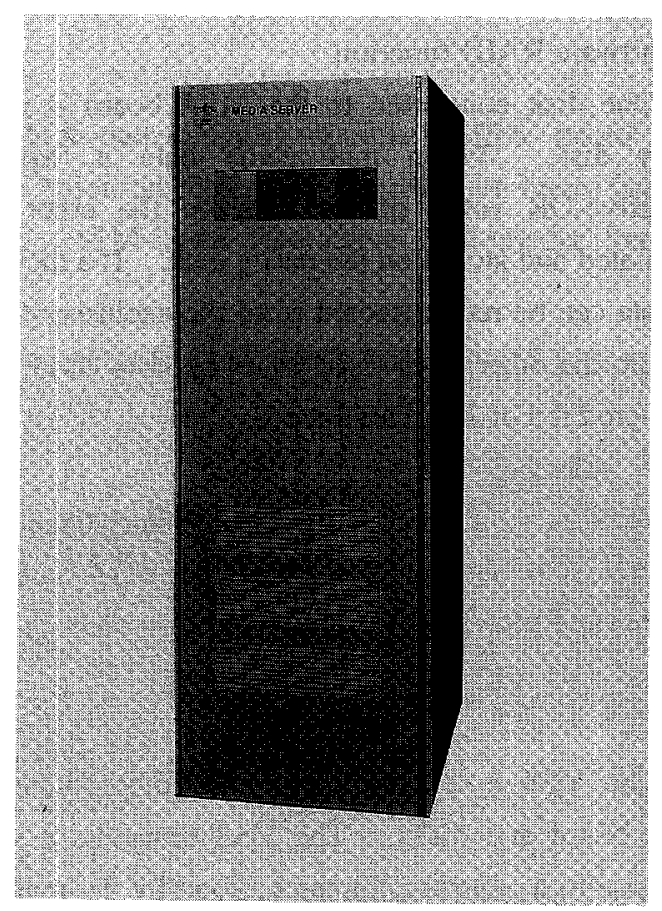

Figure 5. VSS

\section{3) Open architecture}

The system uses an interface conforming to the DAVIC (Digital Audio-Visual Council) control channel specifications for the control commands to the VSS. This provides mutual connectivity with set-top boxes and systems from other manufacturers.

4) Scalability

Since the stream transmission section is provided as a VSS component, high expandability is assured. The number of VSSs in a Fujitsu Media Server can be changed from one to several dozens depending on the purpose and application of the system. When multiple VSSs are used, the application can be used with the same ease as when just a single VSS is used.

5) High data maintainability

The software RAID function of the MSA board enables fast data recovery in case of a disk failure. A disk group technique is used to handle a group of disks as a single unit. This technique prevents a failure in a disk group from affecting other disk groups, enhancing data maintainability.

\section{CONCLUSION}

As described above, the MSA board and the VSS are optimized to store and transmit multimedia data and to enable the concurrent transmission of many streams. A Fujitsu Media Server utilizing the VSS as a storage subsystem provides total cost-efficient support from storage to transmission of videos.

We will develop additional multimedia server systems to provide enhanced cost efficiency. These include systems using servers for public networks, more powerful MSA boards that utilize fiber channels, other high-speed and larger capacity disks, and high-speed network interfaces.

\section{REFERENCE}

[1] K.Nishikawa, et al:: High Performance VOD Server AIMS. GLOBECOM 95 Conference Record, 2. pp. 795 798. 